4. Proof of Theorem 2. Harish-Chandra [1] and others have proved that every Lie algebra over a field of characteristic zero has a faithful representation. Consequently by Lemma $4, \&$ has a faithful representation $x \rightarrow Q_{x}$ whose matrices have elements in an algebraic extension $\Re$ of $\mathfrak{F}$ such that $t\left(Q_{x} Q_{y}\right)=0$ for all $x$ of $\Re$ and all $y$ of $\mathbb{R}$. We now apply another form of Cartan's criterion for solvability which states that if $t\left(A^{2}\right)=0$ for all $A$ in a Lie algebra $\mathfrak{A}$ of linear transformations, than $\mathfrak{A}$ is solvable, and deduce that the ideal $\mathfrak{B}$ of all $\boldsymbol{x}$ of $\mathfrak{Z}$ such that $t\left(Q_{x} Q_{y}\right)=0$ for every $y$ of $\mathfrak{R}$ is solvable. This proves the theorem for we now have $\mathfrak{B}=\mathfrak{R}$ as above.

\title{
REFERENCES
}

1. Harish-Chandra, Faithful representations of Lie algebras, Ann. of Math. vol. 50 (1949) pp. 68-76.

2. - On the radical of a Lie algebra, Proceedings of the American Mathematical Society vol. 1 (1950) pp. 14-17.

3. N. Jacobson, Completely reducible Lie algebras of linear transformations, Proceedings of the American Mathematical Society vol. 2 (1951) pp. 105-113.

EMORY UNIVERSITY

\section{A SUBDIRECT-UNION REPRESENTATION FOR COMPLETELY DISTRIBUTIVE COMPLETE LATTICES}

GEORGE N. RANEY

1. Introduction. In [1], ${ }^{1}$ Garrett Birkhoff makes the following remark: "Tarski has shown that any complete, completely distributive Boolean algebra is isomorphic with the field of subsets of a suitable set. One can also show that any closed sublattice of a direct union of complete chains is a complete, completely distributive lattice. The question is (no. 69), are there any other complete, completely distributive lattices?" This paper will answer Birkhoff's question by proving the following theorem:2

THEOREM A. Every completely distributive complete lattice is iso-

Presented to the Society, October 25, 1952; received by the editors November 11, 1952.

${ }^{1}$ Numbers in brackets refer to the references cited at the end of the paper.

2 Definitions and notations used here conform with those of [3], on which this paper is based. 
morphic with a closed sublattice of the direct union of a family of complete chains.

\section{A characterization of complete distributivity.}

Definition 1 . If $L$ is a partially ordered set and if $M$ is a subset of $L$ such that if $x \in M$ and $y \leqq x$, then $y \in M$, then $M$ is called a semiideal of $L$. Let $R(L)$ denote the complete lattice of semi-ideals of $L$.

Definition 2. If $L$ is a complete lattice and $x \in L$, then let $K(x)$ $=\prod\{M \mid M \in R(L)$ and $x \leqq \cup M\}$.

LEMMA 1. If $L$ is a complete lattice, then

(A) if $x \in L$, then $\cup K(x) \leqq x$;

(B) if $x \in L, y \in L$, and $x \leqq y$, then $K(x) \subset K(y)$;

(C) if $A \subset L$, then $\sum\{K(a) \mid a \in A\}=K(\cup A)$.

Proof. If $x \in L$, then $\{t \mid t \leqq x\} \in R(L)$ and $U\{t \mid t \leqq x\}=x$, so that $K(x) \subset\{t \mid t \leqq x\}$. Therefore $U K(x) \leqq x$. If $x \in L, y \in L$, and $x \leqq y$, then $\{M \mid M \in R(L)$ and $y \leqq U M\} \subset\{M \mid M \in R(L)$ and $x \leqq U M\}$; hence $K(x) \subset K(y)$. If $A \subset L$ and $t \notin \sum\{K(a) \mid a \in A\}$, then for every $a \in A$, $t \notin K(a)$ and one can choose an $M_{a} \in R(L)$ such that $t \notin M_{a}$ and $a$ $\leqq \cup M_{a}$. Then $t \notin \sum\left\{M_{a} \mid a \in A\right\}$. Moreover, $K(\cup A) \subset \sum\left\{M_{a} \mid a\right.$ $\in A\}$, since $\sum\left\{M_{a} \mid a \in A\right\} \in R(L)$ and $U A \leqq \cup\left\{\cup M_{a} \mid a \in A\right\}$ $=\bigcup \sum\left\{M_{a} \mid a \in A\right\}$. Hence $t \notin K(\cup A)$. On the other hand, if $t$ $\in \sum\{K(a) \mid a \in A\}$, then $t \in K(a)$ for some $a \in A$, and since $a \leqq U A$, $t \in K(\cup A)$. Therefore $\sum\{K(a) \mid a \in A\}=K(\bigcup A)$.

LEMMA 2. In order that a complete lattice $L$ be completely distributive it is necessary and sufficient that if $\left\{M_{\gamma} \mid \gamma \in C\right\}$ is a family of semiideals of $L$, then $\cap\left\{U M_{\gamma} \mid \gamma \in C\right\} \leqq \cup \prod\left\{M_{\gamma} \mid \gamma \in C\right\}$.

This follows from Theorem 1 and Lemma 5 of [3].

TheOREM 1. In order that a complete lattice $L$ be completely distributive it is necessary and sufficient that for every $x \in L, U K(x)=x$.

Proof. To prove necessity, let the complete lattice $L$ be completely distributive. If $x \in L$, then $x \leqq \cap\{U M \mid M \in R(L)$ and $x \leqq U M\}$ $\leqq U \prod\{M \mid M \in R(L)$ and $x \leqq U M\}=U K(x)$. This, together with Lemma $1(\mathrm{~A})$, implies that $\cup K(x)=x$ for every $x \in L$.

To prove sufficiency, let $L$ be a complete lattice such that for every $x \in L, U K(x)=x$. If $\left\{M_{\gamma} \mid \gamma \in C\right\}$ is a family of semi-ideals of $L$ and if $t \in K\left(\cap\left\{\cup M_{\gamma} \mid \gamma \in C\right\}\right)$, then for every $\gamma \in C, t \in K\left(\cup M_{\gamma}\right)$, by Lemma $1(\mathrm{~B})$, and $t \in \sum\left\{K(x) \mid x \in M_{\gamma}\right\}$, by Lemma 1(C). For every $\gamma \in C$ one can choose an $x_{\gamma} \in M_{\gamma}$ such that $t \in K\left(x_{\gamma}\right)$ and then $t$ $\leqq U K\left(x_{\gamma}\right)=x_{\gamma}$. Hence $t \leqq n\left\{x_{\gamma} \mid \gamma \in C\right\}$ and, since $\cap\left\{x_{\gamma} \mid \gamma \in C\right\}$ $\in \prod\left\{M_{\gamma} \mid \gamma \in C\right\}, t \in \prod\left\{M_{\gamma} \mid \gamma \in C\right\}$. Therefore $K\left(\cap\left\{\cup M_{\gamma} \mid \gamma \in C\right\}\right)$ 
$\subset \prod\left\{M_{\gamma} \mid \gamma \in C\right\}$. It follows that $\cap\left\{\cup M_{\gamma} \mid \gamma \in C\right\}=U K\left(\cap\left\{\cup M_{\gamma} \mid \gamma\right.\right.$ $\in C\}) \leqq \cup \prod\left\{M_{\gamma} \mid \gamma \in C\right\}$, and, by Lemma $2, L$ is completely distributive.

Definition 3. If $L$ is a complete lattice, let $\rho$ be the binary relation on $L$ defined as follows: $x \rho y$ if and only if $x \in L, y \in L$, and $x \in K(y)$.

Definition 4. If $\sigma$ is a binary relation on a set $X$, let $\sigma \circ \sigma$ be the binary relation on $X$ defined as follows: $x \sigma \circ \sigma y$ if and only if there exists a $z$ such that $x \sigma z$ and $z \sigma y$.

Corollary. If $L$ is a completely distributive complete lattice, then $\rho=\rho \circ \rho$.

Proof. For every $x \in L, K(x)=K(\cup K(x))=\sum\{K(a) \mid a \in K(x)\}$, by Theorem 1 and Lemma $1(\mathrm{C})$. It then follows that $\rho=\rho \circ \rho$.

The nonmodular lattice of five elements is a complete lattice in which $\rho=\rho \circ \rho$ and which is not completely distributive. Hence the converse of the corollary is not true.

3. Relations $\sigma=\sigma \circ \sigma$. Let $X$ be a set and let $\sigma$ be a binary relation on $X$ such that $\sigma=\sigma \circ \sigma$.

Definition 5. If $A \subset X$, let $\phi(A)$ be the set of $x \in X$ such that there exists a $y \in A$ such that $x \sigma y$. Let $L(\sigma)$ be the family $\{\phi(A) \mid A \subset X\}$, partially ordered by set-inclusion.

THEOREM 2. If $\sigma$ is a binary relation on a set $X$ and if $\sigma=\sigma \circ \sigma$, then $L(\sigma)$ is a completely distributive complete lattice. If, in addition, $\sigma$ is reflexive, then $L(\sigma)$ is a complete ring of sets.

Proof. If $\left\{A_{\gamma} \mid \gamma \in C\right\}$ is a family of subsets of $X$, and if $x \in \phi\left(\sum\left\{A_{\gamma} \mid \gamma \in C\right\}\right)$, then there is a $\gamma \in C$ and a $y \in A_{\gamma}$ such that $x \sigma y$. Then $x \in \phi\left(A_{\gamma}\right)$; hence $x \in \sum\left\{\phi\left(A_{\gamma}\right) \mid \gamma \in C\right\}$. This proves that $\phi\left(\sum\left\{A_{\gamma} \mid \gamma \in C\right\}\right) \subset \sum\left\{\phi\left(A_{\gamma}\right) \mid \gamma \in C\right\}$. For every $\gamma \in C, \phi\left(A_{\gamma}\right)$ $C \phi\left(\sum\left\{A_{\gamma} \mid \gamma \in C\right\}\right)$. Therefore $\sum\left\{\phi\left(A_{\gamma}\right) \mid \gamma \in C\right\}=\phi\left(\sum\left\{A_{\gamma} \mid \gamma \in C\right\}\right)$ and $L(\sigma)$ is closed with respect to union. Hence $L(\sigma)$ is a complete lattice, in which joins are unions; that is, $U\left\{\phi\left(A_{\gamma}\right) \mid \gamma \in C\right\}$ $=\sum\left\{\phi\left(A_{\gamma}\right) \mid \gamma \in C\right\}$.

If $A \subset X$ and $x \in \phi(A)$, then there is a $y \in A$ such that $x \sigma y$. Since $\sigma=\sigma \circ \sigma$, there is a $t$ such that $x \sigma t$ and $t \sigma y$. Hence if $x \in \phi(A)$, then there is a $t \in \phi(A)$ such that $x \in \phi(\{t\})$. Therefore $\phi(A) \subset \sum\{\phi(\{t\}) \mid t$ $\in \phi(A)\}$.

If $t \in \phi(A)$ and $M$ is a semi-ideal in $L(\sigma)$ such that $\phi(A) \subset \sum M$, then there exists a $B C X$ such that $\phi(B) \in M$ and $t \in \phi(B)$. Then $\phi(\{t\}) \subset \phi(B)$; hence $\phi(\{t\}) \in M$. Therefore, if $t \in \phi(A)$, then $\phi(\{t\})$ $\in K(\phi(A))$. It follows that $\phi(A) \subset \sum K(\phi(A))=\bigcup K(\phi(A))$. This, 
together with Lemma $1(\mathrm{~A})$, implies that $\phi(A)=\mathrm{U} K(\phi(A))$ for every $A \subset X$. This proves that $L(\sigma)$ is completely distributive.

If, in addition, $\sigma$ is reflexive, then for every $A \subset X, A \subset \phi(A)$. Hence if $\left\{A_{\gamma} \mid \gamma \in C\right\}$ is a family of subsets of $X$, then $\prod\left\{\phi\left(A_{\gamma}\right) \mid \gamma \in C\right\}$ $C \phi\left(\prod\left\{\phi\left(A_{\gamma}\right) \mid \gamma \in C\right\}\right)$. On the other hand, for every $\gamma \in C$, $\phi\left(\prod\left\{\phi\left(A_{\gamma}\right) \mid \gamma \in C\right\}\right) \subset \phi\left(\phi\left(A_{\gamma}\right)\right)=\phi\left(A_{\gamma}\right)$. Therefore, $\prod\left\{\phi\left(A_{\gamma}\right) \mid \gamma\right.$ $\in C\}=\phi\left(\prod\left\{\phi\left(A_{\gamma}\right) \mid \gamma \in C\right\}\right)$, and $L(\sigma)$ is closed with respect to intersection as well as union. In other words, $L(\sigma)$ is a complete ring of sets.

If $\sigma=\sigma \circ \sigma$ and $\sigma$ is reflexive, then $\sigma$ is a quasi-ordering. Theorem 2 shows that the relation between completely distributive complete lattices and relations $\sigma=\sigma \circ \sigma$ is a generalization of the relation between complete rings of sets and quasi-orderings. The latter relation has been studied by G. Birkhoff in [2].

\section{Proof of Theorem A.}

Definition 6. If $\sigma$ is a binary relation on a set $X$, and if $C$ is a subset of $X$ such that if $x \in C$ and $y \in C$, then either $x=y$ or $x \sigma y$ or $y \sigma x$, then $C$ is called a chain in $\sigma$. If $C$ is a chain in $\sigma$ which is not properly contained in any chain in $\sigma$, then $C$ is called a maximal chain in $\sigma$.

It follows from Zorn's Lemma that every chain in $\sigma$ is contained in a maximal chain in $\sigma$.

Let $L$ be a completely distributive complete lattice and let $\Gamma$ be the family of maximal chains in $\rho$. If $C \in \Gamma$ and $a \in L$, let $f(C, a)$ be the set of $t \in C$ such that there exists an $x \in C$ such that toxpa. If $C \in \Gamma$, let $F_{C}=\{f(C, a) \mid a \in L\}$.

For every $x \in L, \sum\{f(C, x) \mid C \in \Gamma\}=K(x)$. For if $t \in K(x)$, then $\{t, x\}$ is a chain in $\rho$, so that there is a $C \in \Gamma$ such that $\{t, x\} \subset C$. Since

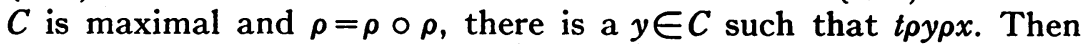
$t \in f(C, x)$. Therefore $K(x) \subset \sum\{f(C, x) \mid C \in \Gamma\}$. On the other hand, if $t \in f(C, x)$ for some $C \in \Gamma$, then $t \in K(x)$. Therefore $\sum\{f(C, x) \mid C$ $\in \Gamma\} \subset K(x)$.

If $a \in L$ and $b \in L$, then either $f(C, a) \subset f(C, b)$ or $f(C, b) \subset f(C, a)$. For if $f(C, a)$ is not contained in $f(C, b)$, then there is a $t \in f(C, a)$ such that $t \notin f(C, b)$. If $y \in f(C, b)$, then neither $t=y$ nor $t \rho y$; otherwise $t \in f(C, b)$. Hence $y \rho t$ and $y \in f(C, a)$. Then $f(C, b) \subset f(C, a)$. Therefore, for every $C \in \Gamma, F_{C}$ is a chain in the relation of set-inclusion on the set of subsets of $C$.

If $C \in \Gamma$ and $A \subset L$, then $\sum\{f(C, a) \mid a \in A\}=f(C, \cup A)$. For $t$ $\in f(C, \cup A)$ if and only if $t \in C$ and there is an $x \in C$ such that $t \rho x \rho \cup A$. By Lemma $1(\mathrm{C}), \operatorname{t\rho x} \rho \cup A$ if and only if there exists an $a \in A$ such that

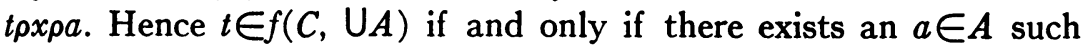
that $t \in f(C, a)$. Therefore $F_{C}$ is closed with respect to union, for every 
$C \in \Gamma$. It follows that for every $C \in \Gamma, F_{C}$ is a complete chain in which, if $F \subset F_{C}, U F=\sum F$ and $\cap F=\sum\left\{f(C, b) \mid b \in L\right.$ and $\left.f(C, b) \subset \prod F\right\}$. If $C \in \Gamma$ and $A \subset L$, then $\cap\{f(C, a) \mid a \in A\}=f(C, \cap A)$. For if $t$ $\in \cap\{f(C, a) \mid a \in A\}$, then there exists a $b \in L$ such that $t \in f(C, b)$ and $f(C, b) \subset \prod\{f(C, a) \mid a \in A\}$. Then $t \in C$ and there exists an $s \in C$ such that $t \rho s \rho b$. Since $\rho=\rho \circ \rho$ and since $C$ is a maximal chain in $\rho$,

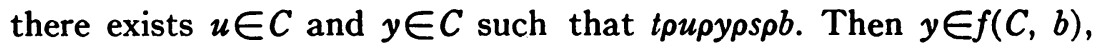
and for every $a \in A, y \in f(C, a)$. Hence $y \leqq a$ for every $a \in A$; so that $y \leqq \cap A$. By Lemma $1(\mathrm{~B}), \operatorname{t\rho u\rho } \cap A$, and $t \in f(C, \cap A)$. Therefore $\cap\{f(C, a) \mid a \in A\} \subset f(C, \cap A)$. On the other hand, $f(C, \cap A)$ $\subset \prod\{f(C, a) \mid a \in A\}$, by Lemma $1(\mathrm{~B})$. Therefore, $f(C, \cap A)$ $\subset \cap\{f(C, a) \mid a \in A\}$.

Let $D$ be the direct union of the family of complete chains $\left\{F_{C} \mid C \in \Gamma\right\} . D$ consists of all functions $\theta: \Gamma \rightarrow \sum\left\{F_{C} \mid C \in \Gamma\right\}$ such that for every $C \in \Gamma, \theta(C) \in F_{C}$. Furthermore $D$ is a complete lattice in which, if $D_{1} \subset D$, then $\left(U D_{1}\right)(C)=U\left\{\theta(C) \mid \theta \in D_{1}\right\}$ and $\left(\cap D_{1}\right)(C)$ $=\cap\left\{\theta(C) \mid \theta \in D_{1}\right\}$, for every $C \in \Gamma$.

For every $a \in L$, let $\theta_{a}$ be the member of $D$ such that for every $C \in \Gamma, \theta_{a}(C)=f(C, a)$. Let $L^{*}=\left\{\theta_{a} \mid a \in L\right\}$. The mapping $a \rightarrow \theta_{a}$ is a one-to-one mapping of $L$ onto $L^{*}$. For if $\theta_{a}=\theta_{b}$, then for every $C \in \Gamma, f(C, a)=f(C, b)$. Then $a=\bigcup K(a)=\bigcup \sum\{f(C, a) \mid C \in \Gamma\}$ $=\bigcup \sum\{f(C, b) \mid C \in \Gamma\}=U K(b)=b$.

If $A \subset L$, then $\cup\left\{\theta_{a} \mid a \in A\right\}=\theta_{\cup A}$. For, if $C \in \Gamma$, then $\left(\cup\left\{\theta_{a} \mid a\right.\right.$ $\in A\})(C)=\bigcup\left\{\theta_{a}(C) \mid a \in A\right\}=\bigcup\{f(C, a) \mid a \in A\}=\sum\{f(C, a) \mid a \in A\}$ $=f(C, \cup A)=\theta_{\cup A}(C)$. Also if $A \subset L$, then $\cap\left\{\theta_{a} \mid a \in A\right\}=\theta_{\cap A}$. For, if $C \in \Gamma$, then $\left(\cap\left\{\theta_{a} \mid a \in A\right\}\right)(C)=\cap\left\{\theta_{a}(C) \mid a \in A\right\}=\cap\{f(C, a) \mid a \in A\}$ $=f(C, \cap A)=\theta_{\cap A}(C)$. It follows that $L^{*}$ is a closed sublattice of $D$, and that the mapping $a \rightarrow \theta_{a}$ is a complete-isomorphism of $L$ onto $L^{*}$.

\section{REFERENCES}

1. G. Birkhoff, Some problems of lattice theory, Proceedings of the International Congress of Mathematicians, Cambridge, U.S.A., 1950, vol. 2, p. 6.

2. G. Birkhoff, On rings of sets, Duke Math. J. vol. 3 (1937) pp. 443-454.

3. G. N. Raney, Completely distritutive complete lattices, Proc. Amer. Math. Soc. vol. 3 (1952) pp. 677-680.

Columbia University 